\title{
Asymmetric oscillatory expansion of a cylindrical plasma
}

A R Karimov, M Y Yu and Lennart Stenflo

\section{Linköping University Post Print}

\section{Tweet}

N.B.: When citing this work, cite the original article.

Original Publication:

A R Karimov, M Y Yu and Lennart Stenflo, Asymmetric oscillatory expansion of a cylindrical plasma, 2013, Journal of Plasma Physics, (79), 6, 1007-1009.

http://dx.doi.org/10.1017/S0022377813000901

Copyright: Cambridge University Press (CUP)

http://www.cambridge.org/uk/

Postprint available at: Linköping University Electronic Press

http://urn.kb.se/resolve?urn=urn:nbn:se:liu:diva-103071 


\title{
Asymmetric oscillatory expansion of a cylindrical plasma
}

\author{
A. R. K A R I MOV V ${ }^{1,2}$, M. Y. Y $\mathrm{U}^{3}$ and L. STEN F L O \\ ${ }^{1}$ Institute for High Temperatures, Russian Academy of Sciences, Izhorskaya 13/19, Moscow 127412, Russia \\ ${ }^{2}$ Department of Electrophysical Facilities, National Research Nuclear University MEPhI, Kashirskoye shosse 31, \\ Moscow 115409, Russia \\ ${ }^{3}$ Institute for Fusion Theory and Simulation, Department of Physics, Zhejiang University, 310027 Hangzhou, China \\ (myyu@zju.edu.cn) \\ ${ }^{4}$ Department of Physics, Linköping University, SE-58183 Linköping, Sweden
}

(Received 24 June 2013; revised 24 June 2013; accepted 18 July 2013; first published online 19 September 2013)

\begin{abstract}
Asymmetric oscillatory expansion of a cylindrical plasma layer into vacuum is investigated analytically by solving the fluid equations of the electrons and ions together with the Maxwell's equations. For the problem considered, it is found that the asymmetrical flow components are strongly affected by the symmetrical components, but not the vice versa.
\end{abstract}

\section{Introduction}

Expansion of a gas or plasma into vacuum is of interest in many areas of research and applications, such as astrophysical explosions, inertial confinement fusion, laser acceleration of charged particles, etc. involving high energy density, as defined in Davidson et al. (2004). Since the problem is intrinsically highly nonlinear, it is traditionally investigated by assuming that the process is self-similar, so that the governing equations can be simplified and solved analytically. Despite the over-simplification, significant and useful solutions have been obtained (see for example Sedov 1959; Gurevich and Pariiskaya 1986; Zel'dovich and Raizer 2002). On the other hand, in reality many expansions are not, or not fully, self-similar (Sedov 1959). Thus, besides solving the more general problems numerically, it is also desirable to search for solutions using analytical methods.

Many paradigm linear partial differential equations in science and engineering can be reduced to ordinary differential equations by using the method of separation of variables (see for example Arfken 1985). On rare occasions, similar separation of variables has also been successfully applied to solving nonlinear evolution equations (Stenflo 1990; Stenflo and Yu 2002; Karimov and Godin 2009). In such schemes, one first makes an Ansatz, usually by intuitive trial and error, on the spatial behavior of the physical variables such that a system of coupled nonlinear ordinary differential equations for the temporal evolution of the latter can be obtained. Recently, we have used this approach to consider the radial expansion of a cylindrical plasma and found that the expansion can be accompanied by intense oscillations as well as energy exchange among the different spatial components. In the present paper, we extend our work to include axial variations that can lead to asymmetry and rotation in the expansion. It is found that in the problem considered, the added asymmetric flow components do not affect the evolution of the symmetric flow components.

Our work is motivated by the following question: in the radial expansion of a cylindrical plasma layer (Karimov et al. 2009a, b), how does flow asymmetry affect the development of the expansion and the accompanying plasma oscillations, and what new flow behavior can appear? In particular, we shall consider the development of the nonlinear oscillations in an axially asymmetric plasma expansion when the vorticity $\Omega_{\varphi}$ is finite (Nijboer et al. 1997; Karimov 2009), or

$$
\Omega_{j \varphi}=\partial_{r} v_{j z}-\partial_{z} v_{j r} \neq 0,
$$

where $\boldsymbol{v}_{j}$ is the velocity of the species $j=i, e$.

\section{Formulation}

We introduce a simple (but not necessarily small) deviation of the originally (Karimov et al. 2009a, b) axially symmetric velocity field by adding a function of $r$ and $t$ to the axial velocity component. Accordingly, we make the Ansatz

$$
\begin{aligned}
& v_{j r}=A_{j}(t) r, \quad v_{j \varphi}=C_{j}(t) r, \quad \text { and } \\
& v_{j z}=B_{j}(t) z+\beta_{j}(t) Q(t, r),
\end{aligned}
$$

$$
\boldsymbol{E}=\varepsilon_{r}(t) \boldsymbol{r} \boldsymbol{e}_{r}+\varepsilon_{\varphi}(t) r \boldsymbol{e}_{\varphi}+\left[\varepsilon_{z}(t) z+\varepsilon_{z r}(t) Q(t, r)\right] \boldsymbol{e}_{z},
$$

where the additional (with respect to that in Karimov et al. 2009a) functions $\beta_{j}(t), \varepsilon_{z r}(t)$, and $Q(t, r)$ allow for asymmetry of the expansion. One can see that we have allowed for an arbitrary function $Q(t, r)$, which can be useful to specific applications and shows that the nonlinear solutions are not unique, as to be expected.

The straightforward but tedious derivation of the resulting evolution equations for the flow components is similar to that of our earlier works (Karimov et al. 2009a, b). Accordingly, in the following we shall directly 
present the ordinary differential equations governing the evolution of the expansion of a cold cylindrical plasma layer.

\section{The evolution equations}

Following Karimov et al. (2009a), one can derive from the cold electron and ion fluid and Maxwell's equations without any approximation the following ordinary differential equations governing the evolution of the asymmetric cylindrical plasma with rotation:

$$
\begin{gathered}
d_{t} n+\left(2 A_{e}+B_{e}\right) n=0, \\
d_{t} A_{e}+A_{e}^{2}-C_{e}^{2}+\varepsilon_{r}+C_{e} B_{z}=0, \\
d_{t} B_{e}+B_{e}^{2}+\varepsilon_{z}=0, \\
d_{t} C_{e}+2 A_{e} C_{e}+\varepsilon_{\varphi}-A_{e} B_{z}=0, \\
d_{t} A_{i}+A_{i}^{2}-C_{i}^{2}-\mu_{i} \varepsilon_{r}-\mu_{i} C_{i} B_{z}=0, \\
d_{t} B_{i}+B_{i}^{2}-\mu_{i} \varepsilon_{z}=0, \\
d_{t} C_{i}+2 A_{i} C_{i}-\mu_{i} \varepsilon_{\varphi}+\mu_{i} A_{i} B_{z}=0, \\
d_{t} \varepsilon_{r}=n\left(A_{e}-A_{i}\right)-\left(2 \varepsilon_{r}+\varepsilon_{z}\right) A_{i}, \\
d_{t} \varepsilon_{\varphi}=n\left(C_{e}-C_{i}\right)-\left(2 \varepsilon_{r}+\varepsilon_{z}\right) C_{i}, \\
d_{t} \varepsilon_{z}=n\left(B_{e}-B_{i}\right)-\left(2 \varepsilon_{r}+\varepsilon_{z}\right) B_{i}, \\
d_{t} B_{z}=-2 \varepsilon_{\varphi} .
\end{gathered}
$$

The evolution of the asymmetric flow components is governed by

$$
\begin{gathered}
Q d_{t} \beta_{j}+\beta_{j} d_{t} Q+A_{j} \beta_{j} r d_{r} Q+\beta_{j} B_{j} Q-\mu_{j} \varepsilon_{z r} Q=0, \\
Q d_{t} \varepsilon_{z r}+\varepsilon_{z r} d_{t} Q+n\left(\beta_{i}-\beta_{e}\right)+\left(2 \varepsilon_{r}+\varepsilon_{z}\right) \beta_{i}=0,
\end{gathered}
$$

where we recall that $Q$ can be a function of $t$ and $r$.

Eliminating $d_{t} Q$ from (3.12) with the help of (3.13), we get

$$
\begin{aligned}
& \varepsilon_{z r}\left[d_{t} \beta_{j}+\beta_{j} B_{j}-\mu_{j} \varepsilon_{z r}\right]+\beta_{j}\left[n\left(\beta_{e}-\beta_{i}\right)-\left(2 \varepsilon_{r}+\varepsilon_{z}\right)\right. \\
& \left.\quad \times \beta_{i}-d_{t} \varepsilon_{z r}\right]+A_{j} \beta_{j} \varepsilon_{z r} \frac{r}{Q} d_{r} Q=0 .
\end{aligned}
$$

As mentioned, the function $Q(t, r)$ is a free parameter. It can be chosen such that it becomes decoupled from the other variables. We can in fact separate the $t$ and $r$ dependence of $Q(t, r)$ by setting $Q(t, r)=q(t) r^{\sigma}$, where $\sigma$ is an arbitrary constant and $q(t)$ is an arbitrary function of $t$ only. Then (3.14) becomes

$$
\varepsilon_{z r} d_{t} \beta_{j}=\left[d_{t} \varepsilon_{z r}+\left(\beta_{i}-\beta_{e}\right) n+\left(2 \varepsilon_{r}+\varepsilon_{z}\right)\right.
$$

$$
\left.\times \beta_{i}-\varepsilon_{z r}\left(B_{j}+\sigma A_{j}\right)\right] \beta_{j}+\mu_{j} \varepsilon_{z r}^{2},
$$

and (3.13) becomes

$$
\varepsilon_{z r} d_{t} q=\left[n\left(\beta_{e}-\beta_{i}\right)-\left(2 \varepsilon_{r}+\varepsilon_{z}\right) \beta_{i}-d_{t} \varepsilon_{z r}\right] q .
$$

We can further set $q(t)=q_{0} e^{t / \tau}$, where $q_{0}$ and $\tau$ are constants, so that

$$
d_{t} \varepsilon_{z r}=n\left(\beta_{e}-\beta_{i}\right)-\left(2 \varepsilon_{r}+\varepsilon_{z}\right) \beta_{i}-\varepsilon_{z r} / \tau .
$$

Substitution of (3.17) into (3.15) then leads to

$$
d_{t} \beta_{j}=-\left(1 / \tau+B_{j}+\sigma A_{j}\right) \beta_{j}+\mu_{j} \varepsilon_{z r},
$$

which completes the set of equations governing the evolution of the asymmetric flow expansion. One can see that the symmetric flow components $A_{j}$, etc. can affect the asymmetric flow components $\beta_{j}$, but not the vice versa: $\beta_{j}$ does not affect $A_{j}$, etc. Accordingly, the results on the evolution of the symmetric flow components obtained in Karimov et al. (2009a) remain valid also for the asymmetric expansion.

\section{Discussion and conclusion}

It is remarkable that although the symmetric flow components can strongly affect the asymmetric ones, yet the latter do not affect the former. That is, the flow asymmetry does not affect the evolution of the symmetric flow components, and the behavior of the symmetric part of the flow as discussed in detail in Karimov et al. (2009a) is thus also applicable even when asymmetric flow is introduced. However, the latter also depend on the constants $\sigma$ and $\tau$, which are determined externally by the physical situation (such as the presence of external drive) and characterize the space and time scales of the evolution of the asymmetric components of the flow.

It is of interest to note that the oscillations can be considered as energy and momentum redistribution from the flow expansion. Such energy and momentum redistribution can prevent the occurrence of shockor soliton-like structures, or singularities, during the expansion, whose speed can increase with time as the densities decrease. We also note that depending on the signs of $\sigma$ and $\tau$ as determined by the initial condition, the flow asymmetry can increase with $t$ and $r$.

We emphasize that, as for most nonlinear problems, the conclusion here is not unique and other flow behavior can also exist. Nevertheless, our results illustrate an unusual nonlinear plasma flow property that could be useful to interpreting unexpected phenomena, such as weak- or de-coupling among the degrees of freedom, in high energy-density astrophysical and laser-induced plasma expansions and explosions, as well as other areas (Gurevich and Pariiskaya 1986; Bartel et al. 1991; Blondin et al. 1996; Zel'dovich and Raizer 2002; Mora 2003; Davidson et al. 2004; Kaladze et al. 2007; Shukla and Eliasson 2009; Meliani and Keppens 2010; Mamun and Shukla 2011). 


\section{Acknowledgements}

This work is supported by the Open Fund of the State Key Laboratory of High Field Laser Physics at SIOM, the Ministry of Science and Technology of China (2011GB105000), and the National Natural Science Foundation of China (10835003).

\section{References}

Arfken, G. 1985 Mathematical Methods for Physicists. New York: Academic Press.

Bartel, N. et al. 1991 Nature 350, 212.

Blondin, J., Lundqvist, P. and Chevalier, R. 1996 Astrophys. J. 472, 257.

Davidson, R. C. et al. 2004 Frontiers For Discovery In High Energy Density Physics, http://www.whitehouse.gov/ sites/default/files/hedpreport.pdf

Gurevich, A. V. and Pariiskaya, L. V. 1986 Reviews of Plasma Physics, Vol. 10. New York: Consultants Bureau.
Kaladze, T. D., Wu, D. J., Pokhotelov, O. A., Sagdeev, R.Z. Stenflo, L. and Shukla, P. K. 2007 J. Plasma Phys. 73, 131.

Karimov, A. R. 2009 J. Plasma Phys. 75, 817.

Karimov, A. R. and Godin, S. M. 2009 Phys. Scr. 80, 035503.

Karimov, A. R., Stenflo, L. and Yu, M. Y. 2009a Phys. Plasmas 16, 062313.

Karimov, A. R., Stenflo, L. and Yu, M. Y. 2009b Phys. Plasmas 16, 102303.

Mamun A. A. and Shukla, P. K. 2011 J. Plasma Phys. 77, 437.

Meliani, Z. and Keppens R. 2010 Astro. Astrophys. 520, L3.

Mora, P. 2003 Phys. Rev. Lett. 90, 185002.

Nijboer, J., Lifschitz, A. E. and Goedbloed, J. P. 1997 J. Plasma Phys. 58, 101.

Sedov, L. 1959 Similarity and Dimensional Methods in Mechanics. New York: Academic Press.

Shukla P. K. and Eliasson, B. 2009 Rev. Mod. Phys. 81, 25.

Stenflo, L. 1990 Phys. Scr. 41, 643.

Stenflo, L. and Yu, M. Y. 2002 Phys. Plasmas 9, 5129.

Zel'dovich, Y.B. and Raizer, Y.P. 2002 Physics of Shock Waves and High-Temperature Hydrodynamic Phenomena. Mineola, N. Y.: Dover. 